\title{
STRUCTURE OF SET OF SYMMETRIES FOR HYPERBOLIC SYSTEMS OF LIOUVILLE TYPE AND GENERALIZED LAPLACE INVARIANTS
}

\author{
S.YA. STARTSEV
}

\begin{abstract}
The present paper is devoted to hyperbolic systems consisting of $n$ partial differential equations and possessing symmetry drivers, i.e., differential operators mapping any function of one independent variable into a symmetry of the corresponding system. The presence of the symmetry drivers is a feature of the Liouville equation and similar systems. The composition of a differential operator with a symmetry driver is a symmetry driver again if the coefficients of the differential operator belong to the kernel of a total derivative. We prove that the entire set of the symmetry drivers is generated via the above compositions from a basis set consisting of at most $n$ symmetry drivers whose sum of orders is the smallest possible.

We also prove that if a system admits a symmetry driver of order $k-1$ and generalized Laplace invariants are well-defined for this system, then the leading coefficient of the symmetry driver belongs to the kernel of the $k$ th Laplace invariant. Basing on this statement, after calculating the Laplace invariants of a system, we can obtain the lower bound for the smallest orders of the symmetry drivers for this system. This allows us to check whether we can guarantee that a particular set of the drivers is a basis set.
\end{abstract}

Keywords: higher symmetries, symmetry drivers, nonlinear hyperbolic partial differential systems, Laplace invariants, Darboux integrability.

Mathematics Subject Classification: 37K05, 37K10, 35L51

\section{INTRODUCTION}

We consider a system of partial differential equations

$$
u_{x y}=F\left(x, y, u, u_{x}, u_{y}\right),
$$

where $u=\left(u^{1} ; u^{2} ; \ldots ; u^{n}\right)$ and $F=\left(F^{1} ; F^{2} ; \ldots ; F^{n}\right)$ are $n$-dimensional vectors and $u$ is a function of variables $x$ and $y$. Since in the present paper all relations are considered on solutions of this system, we can exclude all mixed partial derivatives of $u$ by means of system (1) and its differential implications. This is why without loss of generality we can regard all local objects like symmetries and the coefficients of differential operators as depending on $x, y, u$ and its pure derivatives $u_{i}:=\partial^{i} u / \partial x^{i}, \bar{u}_{j}:=\partial^{j} u / \partial y^{j}$. In what follows we employ the notation $g[u]$ for stressing the fact that $g$ is a function of finitely many aforementioned variables. By $D_{x}$ and $D_{y}$ we denote total derivatives in $x$ and $y$ due to system (1).

In the scalar case, as $n=1$, one of the most known nonlinear equation of form (1) is the Liouville equation $u_{x y}=e^{u}$. It possesses many interesting properties and in particular, as it was shown in work [1], it admits differential operators $\sigma=D_{x}+u_{x}$ and $\bar{\sigma}=D_{y}+u_{y}$ mapping

S.Ya. Startsev, Structure of Set of Symmetries for hyperbolic systems of Liouville type AND GENERALIZED LAPLACE INVARIANTS.

(C)STARTSEV S.YA. 2018.

Submitted July 17, 2018. 
ker $D_{y}$ and ker $D_{x}$ into solutions $f$ of linearized Liouville equation $D_{x} D_{y}(f)=e^{u} f$. In other words, $\sigma(g)$ and $\bar{\sigma}(\bar{g})$ are symmetries of the Liouville equation for all functions $g[u] \in \operatorname{ker} D_{y}$, $\bar{g}[u] \in \operatorname{ker} D_{x}$, for instance, for all functions depending on $x$ and $y$ only.

Many integrable systems of form (1) possess a similar property. For instance, we consider the system

$$
u_{x y}^{1}=\frac{u^{2} u_{x}^{1} u_{y}^{1}}{u^{1} u^{2}+c}, \quad u_{x y}^{2}=\frac{u^{1} u_{x}^{2} u_{y}^{2}}{u^{1} u^{2}+c},
$$

where $c$ is a non-zero constant. This system is a degenerate case of the Pohlmeyer-Lund-Regge system and the operators

$$
\sigma_{1}=\left(\begin{array}{c}
u_{x}^{1} \\
u_{x}^{2}
\end{array}\right), \quad \sigma_{2}=\left(\begin{array}{c}
\frac{u_{x}^{1}}{w} \\
0
\end{array}\right) D_{x}+\left(\begin{array}{c}
-u^{1} \\
u^{2}
\end{array}\right)
$$

where $\omega=u_{x}^{1} u_{x}^{2} /\left(u^{1} u^{2}+c\right)$, map each scalar function in ker $D_{y}$ into a symmetry of this system. In what follows, such operators mapping the kernels of $D_{y}$ and $D_{x}$ into the symmetries of system (1) are called respectively drivers of $x$ - and $y$-symmetries. A more careful definition will be given later and the examples of systems admitting the drivers can be found, in particular, in [2]-[5].

By the symbol o we denote the operator of composition of operators. It is easy to see that if an operator $S$ is a driver of $x$-symmetries, then $S \circ \Omega$ is also a driver of $x$-symmetries for each operator $\Omega=\sum_{i=0}^{m} w_{i}[u] D_{x}^{i}$ such that the scalar functions $w_{i}$ lie in the kernel of $D_{y}$. A similar fact is true for the drivers of $y$-symmetries. Thus, if system (1) admits the drivers of symmetries, there exist infinitely many of such drivers and there arises an issue how to describe briefly this set: whether it is possible to generate this entire set by means of the aforementioned composition of finitely many basis drivers of symmetries? And if so, then how many drivers of the symmetries is needed for this basis set; whether, for instance, it is sufficient to have $n$ drivers in each of the characteristics or there can be more of them? In the present work we answer these questions. Namely, we prove that the set of the drivers of the symmetries is generated by the basis set consisting of at most $n$ drivers of the symmetries, the sum of whose orders is the smallest possible.

We also prove that if system (1) admits a driver of symmetries of order $k-1$ and the generalized Laplace invariants are well-defined for them, then the leading coefficient of the driver of symmetries belongs to the kernel of $k$ th Laplace invariant. Basing on this statement, we can calculate the Laplace invariants for system (1) and to obtain the lower bound for the minimal orders of the drivers of symmetries for this system and to check whether we can ensure that a given set of drivers is basis. It should be noted that the estimates for the lower orders of the drivers of symmetries obtained by means of Laplace invariants turn out to be sharp in all examples the author knows; that is, they provide the exact minimums of the orders.

\section{StruCture of DRIVERS OF SyMmetries}

The symbol $\top$ stands for the matrix transposition. Given a scalar function $g$ and a vector $z=\left(z^{1}, z^{2}, \ldots, z^{n}\right)^{\top}$, by $g_{z}=\partial g / \partial z$ we denote the row $\left(\partial g / \partial z^{1}, \partial g / \partial z^{2}, \ldots, \partial g / \partial z^{n}\right)$. If $g$ is a vector function $\left(g^{1}, g^{2}, \ldots, g^{\ell}\right)^{\top}$, by $g_{z}$ we denote the matrix with the rows $g_{z}^{1}, \ldots, g_{z}^{\ell}$. In view of these standard notations, the total derivatives $D_{x}$ and $D_{y}$ for each scalar function $g[u]$ are 
given by the formulae

$$
\begin{aligned}
& D_{x}(g)=\frac{\partial g}{\partial x}+\frac{\partial g}{\partial u} u_{1}+\sum_{i=1}^{\infty}\left(\frac{\partial g}{\partial u_{i}} u_{i+1}+\frac{\partial g}{\partial \bar{u}_{i}} D_{y}^{i-1}(F)\right), \\
& D_{y}(g)=\frac{\partial g}{\partial y}+\frac{\partial g}{\partial u} \bar{u}_{1}+\sum_{i=1}^{\infty}\left(\frac{\partial g}{\partial \bar{u}_{i}} \bar{u}_{i+1}+\frac{\partial g}{\partial u_{i}} D_{x}^{i-1}(F)\right) .
\end{aligned}
$$

The result of applying $D_{x}$ and $D_{y}$ to the vectors and matrices is defined component-wise. In the above formulae and hereinafter the zero power of each differentiation is let to be the identity mapping.

Definition 1. A vector function $f=\left(f^{1}[u], f^{2}[u], \ldots, f^{n}[u]\right)^{\top}$ is called symmetry of system (1) if $L(f)=0$, where

$$
L=D_{x} D_{y}-F_{u_{x}} D_{x}-F_{u_{y}} D_{y}-F_{u}
$$

Definition 2. The differential operator

$$
\sigma=\sum_{i=0}^{k} \varsigma_{i}[u] D_{x}^{i}, \quad \varsigma_{k} \neq 0, \quad k \geqslant 0,
$$

where $\varsigma_{i}$ are $n$-dimensional vectors is called driver of $x$-symmetries for system (1) if $\sigma(g)$ is a symmetry of this system for each scalar function $g[u] \in \operatorname{ker} D_{y}$. The vector $\varsigma_{k}$ is called separant for driver of symmetries (5), and the number $k$ is called the order of this driver. We shall say that the drivers of the symmetries $\sigma_{1}, \sigma_{2}, \ldots, \sigma_{r}$ are mutually independent if the matrix of size $n \times r$ formed by its separants has the rank $r$.

In the same way we define the drivers of $y$-symmetries: it is sufficient to swap $x$ and $y$ in Definition 2, Employing the symmetricity of formula (1) with respect to the permutation $x \leftrightarrow y$, below we provide only one of two symmetric definitions and statements.

Lemma 1. If operator (5) is a driver of $x$-symmetries, then its coefficients $\varsigma_{i}$ are independent of $\bar{u}_{j}$ for all $j>0$, and its separant $\varsigma_{k}$ lies in the kernel of $D_{y}-F_{u_{x}}$.

Proof. By the definition of a driver of $x$-symmetries, the relation holds $L(\sigma(g))=0$ for each function $g(x)$. Calculating the coefficients at $i$ th derivative of $g$ with respect to $x$ in this identity and taking into the consideration that the function $g$ is arbitrary, we arrive at the following chain of relations:

$$
\begin{aligned}
& \left(D_{y}-F_{u_{x}}\right)\left(\varsigma_{k}\right)=0, \\
& \left(D_{y}-F_{u_{x}}\right)\left(\varsigma_{i-1}\right)=-L\left(\varsigma_{i}\right), \quad 0<i \leqslant k .
\end{aligned}
$$

By the first relation we see that $\varsigma_{k}$ can not depend on the derivatives of $u$ with respect to $y$, while by the second relation that $\left(\varsigma_{i-1}\right)_{\bar{u}_{j}}=0$ for all $j>0$ if $\varsigma_{i}$ is independent of the derivatives of $u$ with respect to $y$.

Definition 3. A set of the drivers of $x$-symmetries $\sigma_{1}, \sigma_{2}, \ldots, \sigma_{r}$ is called basis for system (1) if the following conditions hold:

1) the drivers in this set are mutually independent;

2) system (1) has no set of mutually independent drivers of $x$-symmetries containing more than $r$ drivers;

3) the sum of orders of the drivers in the set is minimal among all sets of $r$ mutually independent drivers of $x$-symmetries of system (1). 
Since there can be at most $n$ mutually independent drivers, Condition 2) in Definition 3 holds immediately in the case $r=n$. But the latter case is not the only possible and, generally speaking, $r$ can be less than $n$. For instance, the system

$$
u_{x y}^{i}=\sum_{j=1}^{n} A_{j}^{i} \mathrm{e}^{u^{j}}, \quad i=1,2, \ldots, n,
$$

admits the driver of $x$-symmetries $(1,1, \ldots, 1)^{\top} D_{x}+u_{x}$ for all values of the constants $A_{j}^{i}$, but in the general situation, it seems to admit no drivers of symmetries with separants different from the vectors of form $(c[u], c[u], \ldots, c[u])^{\top}$. If the latter is true, then, except some special cases, we have $r=1$ for this system.

By Definition 3 we obviously see that the basis set of drivers exists for each system (1) admitting the drivers of symmetries. This is why the following theorem can be applied to each such system.

Theorem 1. Let the set of drivers of $x$-symmetries $\sigma_{1}, \sigma_{2}, \ldots, \sigma_{r}$ is basis for system (1). Then each driver of $x$-symmetries $S$ of this system can be written as

$$
S=\sum_{i=1}^{r} \sigma_{i} \circ \Omega_{i},
$$

where $\Omega_{i}$ are some operators of form $\sum_{j=0}^{q_{i}} w_{i j}[u] D_{x}^{j}$ such that $q_{i} \geqslant 0$ and the scalar functions $w_{i j}$ belong to $\operatorname{ker} D_{y}$.

Proof. We denote the orders of the operators $\sigma_{1}, \sigma_{2}, \ldots, \sigma_{r}$ by $p_{1}, p_{2}, \ldots, p_{r}$, respectively. Without loss of generality we can assume that these operators are arranged so that their orders ascend, that is, $p_{1} \leqslant p_{2} \leqslant \ldots \leqslant p_{r}$. By $\varsigma_{i}$ we denote the separant of the driver $\sigma_{i}$, while $\xi$ and $p$ stand for the separant and order of the driver $S$.

Let us describe the procedure of successive decreasing of the order of $S$ by means of basis drivers under the assumption $p \geqslant p_{r}$; if this assumption fails, we just skip the first steps in this procedure and proceed to the steps described in the next paragraphs.

If $p \geqslant p_{r}$, we can write $\xi=\sum_{i=1}^{r} \tilde{w}_{i}[u]_{\varsigma_{i}}$ by Statement 2 in Definition 3 . Taking into consideration Lemma 1, we obtain

$$
\sum_{i=1}^{r} D_{y}\left(\tilde{w}_{i}\right) \varsigma_{i}=\left(D_{y}-F_{u_{x}}\right)(\xi)=0
$$

and $D_{y}\left(\tilde{w}_{i}\right)=0$ by the linear independence of the vectors $\varsigma_{i}$. This is why the operator

$$
\tilde{S}=S-\sum_{i=1}^{r} \sigma_{i} \circ \tilde{w}_{i} D_{x}^{p-p_{i}}
$$

is a driver of $x$-symmetries (or it just vanishes) and it has the order less than $p$. If the order $\tilde{S}$ is greater than or equal to $p_{r}$, we repeat the described procedure once again applying it $\tilde{S}$ and so forth. Finally, after several steps of the mentioned procedure we either express $S$ as (7) or we represent just several higher order terms of the operator $S$ in form (7) and we obtain the driver of symmetries

$$
\hat{S}=S-\sum_{i=1}^{r} \sigma_{i} \circ \sum_{j=0}^{p-p_{i}} \hat{w}_{i j}[u] D_{x}^{j}, \quad \hat{w}_{i j} \in \operatorname{ker} D_{y}
$$

of an order $\hat{p}<p_{r}$.

Assume that $p_{\ell} \leqslant \hat{p}<p_{\ell+1}$. By Statement 2) of Definition 3, we can write out the separant $\hat{\xi}$ of the driver $\hat{S}$ as $\hat{\xi}=\sum_{i=1}^{r} \bar{w}_{i}[u] \varsigma_{i}$. If $\bar{w}_{i} \neq 0$ for some $i>\ell$, we can replace $\sigma_{i}$ by $\hat{S}$ and obtain 
a new set of $r$ mutually independent drivers with the sum of orders less than $\sum_{j=1}^{r} p_{j}$. But this contradicts Statement 3) in Definition 3. This is why $\bar{w}_{i}=0$ for all $i>\ell$ and $\hat{\xi}=\sum_{i=1}^{\ell} \bar{w}_{i}[u] \varsigma_{i}$. In view of this, we can repeat the aforementioned procedure of order reducing for the driver of symmetries until we make this order less than $p_{1}$.

Repeating the arguing in the previous paragraph in the case $\hat{p}<p_{1}$, we obtain that the driver of the symmetries has an order less than $p_{1}$ if and only if its separant is equal to zero and this contradicts the definition of the driver of the symmetries. Thus, the rest obtained after reduction of the order to that less than $p_{1}$ by the aforementioned procedure, should be zero. And this means that $S$ can be expressed in form (7).

\section{Generalized Laplace transformation FOR the DRIVERS OF SYMmetries}

It is easy to see that operator (4) can be represent as

$$
L=\left(D_{x}-F_{u_{y}}\right) \circ\left(D_{y}-F_{u_{x}}\right)-H_{1},
$$

where $H_{1}=F_{u_{y}} F_{u_{x}}+F_{u}-D_{x}\left(F_{u_{x}}\right)$. Starting with $H_{1}$, we can construct matrices $a_{i}[u], H_{i}[u]$ of size $n \times n$, defined by the recurrent formulae

$$
\begin{aligned}
& D_{y}\left(H_{i} H_{i-1} \ldots H_{1}\right)+H_{i} H_{i-1} \ldots H_{1} F_{u_{x}}+a_{i} H_{i} H_{i-1} \ldots H_{1}=0, \\
& H_{i+1}=D_{x}\left(a_{i}\right)-\left[F_{u_{y}}, a_{i}\right]+D_{y}\left(F_{u_{y}}\right)+H_{i} .
\end{aligned}
$$

Identities (8) - (9) are obtained by straightforward extension of the formulae for the Laplace invariants $H_{i}$ in the scalar case described, for instance, in [6], to the case of systems; the formulae in the scalar case appropriate also for the case of systems were written out, for instance, in Introduction in work [5]. It was mentioned in work [7] that at least for some well-known systems the well-defined objects are not $H_{i}$ but the matrices $Y_{i}=H_{i} H_{i-1} \ldots H_{1}$. This is why we call the matrices $Y_{i}$ generalized Laplace $y$-invariants of system (1).

In the case $\operatorname{det}\left(Y_{i}\right)=0$ equation (8) can have no solution $a_{i}$ but if it exists, then $a_{i}$ is not unique. Hence, the matrix $H_{i+1}$ and the Laplace invariant $Y_{i+1}$ are generally speaking ill-defined: they may be absent or can be defined in a non-unique way. However, at least in certain interesting cases the generalized Laplace invariants $Y_{i}$ exist and uniquely defined by formulae (8)-(9). For instance, as it was shown in [8, 9], all systems of form (6) are among such cases, for which $A_{j}^{i}$ is a Cartan matrix of a simple Lie algebra.

By formulae (9) we see easily that $Y_{i+1}$ is independent of the choice of $a_{i}$ if and only if $\left(D_{x}+F_{u_{y}}^{\top}\right)\left(\operatorname{ker} Y_{i}^{\top}\right) \subset \operatorname{ker} Y_{i}^{\top}$. This means, see [7, Thm. 8] or [10, Prop. 3], that there exists a matrix $B_{i}[u]$ of size $n \times n$ satisfying the operator relation

$$
\left(D_{x}-F_{u_{y}}\right) \circ Y_{i}=Y_{i} \circ\left(D_{x}+B_{i}\right) .
$$

Equation (8) can be written as the operator identity

$$
\left(D_{y}+a_{i}\right) \circ Y_{i}=Y_{i} \circ\left(D_{y}-F_{u_{x}}\right) .
$$

In what follows we assume that the generalized Laplace $y$-invariants of system (1) exist and defined uniquely, that is, the relations (10), (11) hold for all $Y_{i}$, which we shall use for further arguing.

We introduce the operators

$$
\begin{aligned}
& l_{i}=\left(D_{x}-F_{u_{y}}\right) \circ\left(D_{y}+a_{i}\right)-H_{i+1}, \\
& L_{i}=\left(D_{x}-F_{u_{y}}\right) \circ Y_{i} \circ\left(D_{y}-F_{u_{x}}\right)-Y_{i+1} .
\end{aligned}
$$


Denoting $-F_{u_{x}}$ by $a_{0}$ and letting $Y_{0}$ to be the unit matrix of size $n \times n$, we can assume that $l_{0}=L_{0}=L$. It follows from (11) that

$$
\begin{aligned}
& L_{i}=l_{i} \circ Y_{i}, \\
& \left(D_{y}+a_{i+1}\right) \circ L_{i}=\left[\left(D_{y}+a_{i+1}\right) \circ\left(D_{x}-F_{u_{y}}\right)-H_{i+1}\right] \circ Y_{i} \circ\left(D_{y}-F_{u_{x}}\right) .
\end{aligned}
$$

In view of (9), the latter identity can be written as

$$
\left(D_{y}+a_{i+1}\right) \circ L_{i}=l_{i+1} \circ Y_{i} \circ\left(D_{y}-F_{u_{x}}\right) .
$$

Theorem 2. Assume that for system (1) there exist generalized Laplace $y$-invariants $Y_{q}$ for all positive $q \leqslant i+2$ and the Laplace invariant $Y_{i+2}$ is independent of the choice of solution $a_{i+1}$ of equation (8). Suppose also that for this system there exist $n$-dimensional vectors $\alpha_{j}[u]$ such that $\alpha_{m} \neq 0, m>0$, and the operator $P_{i}=\sum_{j=0}^{m} \alpha_{j} D_{x}^{j}$ maps each scalar function from the kernel of $D_{y}$ into the kernel of $L_{i}$.

We denote by $P_{i+1}$ the operator $P_{i+1}=\sum_{j=0}^{m-1} \hat{\alpha}_{j}[u] D_{x}^{j}, \hat{\alpha}_{m-1}=\alpha_{m}$ obtained by dividing the operator $P_{i}$ by the operator $D_{x}+B_{i+1}$, namely, such that

$$
P_{i}=\left(D_{x}+B_{i+1}\right) \circ P_{i+1}+z[u],
$$

where $B_{i+1}$ is determined by formula (10), and $z$ is an $n$-dimensional vector. Then $P_{i+1}$ maps each scalar function in the kernel of $D_{y}$ into the kernel of $L_{i+1}$, and the remainder $z$ of division of $P_{i}$ by $D_{x}+B_{i+1}$ belongs to the kernel of $Y_{i+1}$.

Proof. Let $L_{i}\left(P_{i}(w)\right)=0$ for each scalar function $w[u]$ belonging to ker $D_{y}$. Then, by (13), the identity holds:

$$
l_{i+1}\left(Y_{i}\left(D_{y}-F_{u_{x}}\right)\left(P_{i}(w)\right)\right)=0,
$$

that is, the operator $\hat{P}_{i}=\sum_{j=0}^{m} \beta_{j} D_{x}^{j}$, where $\beta_{j}=Y_{i}\left(D_{y}-F_{u_{x}}\right)\left(\alpha_{j}\right)$, maps the kernel of $D_{y}$ into the kernel of $l_{i+1}$.

On the other hand, repeating the arguing in the proof of Lemma 1, we obtain that the condition $L_{i}\left(P_{i}(w)\right)=0$ is equivalent to the chain of relations

$$
\begin{aligned}
& \beta_{m}=0, \\
& \beta_{j-1}=Y_{i+1} \alpha_{j}-\left(D_{x}-F_{u_{y}}\right)\left(\beta_{j}\right), \quad j=\overline{1, m} \\
& 0=Y_{i+1} \alpha_{0}-\left(D_{x}-F_{u_{y}}\right)\left(\beta_{0}\right) .
\end{aligned}
$$

The second of these formulae for $j=m$ gives $\beta_{m-1}=Y_{i+1} \alpha_{m}$. Employing this and also the fact that thanks to $(10)$, the operator $D_{x}-F_{u_{y}}$ preserves the image of $Y_{i+1}$, by induction in $k$ we can show that $\beta_{m-k}$ lies in the image of $Y_{i+1}$ for all $k=\overline{0, m}$.

Thus, $\beta_{j}=Y_{i+1} \hat{\alpha}_{j}$. Then, taking into consideration (10), we can rewrite the formulae (15) as

$$
\begin{aligned}
& Y_{i+1} \hat{\alpha}_{m}=0, \\
& Y_{i+1} \hat{\alpha}_{j-1}=Y_{i+1}\left(\alpha_{j}-\left(D_{x}+B_{i+1}\right)\left(\hat{\alpha}_{j}\right)\right), \quad j=\overline{1, m} \\
& 0=Y_{i+1}\left(\alpha_{0}-\left(D_{x}+B_{i+1}\right)\left(\hat{\alpha}_{0}\right)\right) .
\end{aligned}
$$

Since $\hat{\alpha}_{j}$ are defined up to the kernel of $Y_{i+1}$, we can choose $\hat{\alpha}_{m}=0, \hat{\alpha}_{m-1}=\alpha_{m}$, and other $\hat{\alpha}_{j}$ can be introduced by the formulae

$$
\hat{\alpha}_{j-1}=\alpha_{j}-\left(D_{x}+B_{i+1}\right)\left(\hat{\alpha}_{j}\right) .
$$

Such choice $\hat{\alpha}_{j}$ ensures that the operator $P_{i+1}=\sum_{j=0}^{m-1} \hat{\alpha}_{j} D_{x}^{j}$ satisfies the relation (14), and the remainder $z$ is defined by the formula $z=\alpha_{0}-\left(D_{x}+B_{i+1}\right)\left(\hat{\alpha}_{0}\right)$ and by the latter identity in (16), $Y_{i+1} z=0$. 
By construction, the operator $P_{i+1}$ is related with the operator $\hat{P}_{i}$ by the relation $Y_{i+1} P_{i+1}=$ $\hat{P}_{i}$ and the operator $\hat{P}_{i}$ maps the kernel of $D_{y}$ into the kernel of $l_{i+1}$. This is why the operator $P_{i+1}$ maps the kernel of $D_{y}$ into the kernel of $l_{i+1} \circ Y_{i+1}$. But by (12), we have $l_{i+1} \circ Y_{i+1}=L_{i+1}$.

Corollary 1. Assume that system (1) admits the driver of $x$-symmetries of $k$ th order with separant $\xi$ and the generalized Laplace $y$-invariants $Y_{q}$ exist and are well-defined for this system for all positive $q \leqslant k+1$. Then $Y_{i} \xi=0$ for all $i>k$, for which there exist the Laplace invariants $Y_{i}$.

Proof. Applying Theorem 2 multiple times, we obtain that $L_{k}(\xi f(x))=0$ for each scalar function $f$. The latter identity is equivalent to the condition $Y_{k+1} \xi=0$ by Lemma 1. It remains to observe that $\operatorname{ker} Y_{i} \subset \operatorname{ker} Y_{i+1}$ for all $i$, for which the Laplace invariant $Y_{i+1}$ exists.

Example 1. As an example, we consider system (2). By straightforward calculations we get

$$
Y_{1}=H_{1}=\frac{c}{\left(u^{1} u^{2}+c\right)^{2}}\left(\begin{array}{cc}
-u_{y}^{1} u_{x}^{2} & u_{x}^{1} u_{y}^{1} \\
u_{x}^{2} u_{y}^{2} & -u_{x}^{1} u_{y}^{2}
\end{array}\right) .
$$

It is easy to see that the kernel of $Y_{1}$ consists of the vectors collinear to the vector $\left(u_{x}^{1}, u_{x}^{2}\right)^{\top}$. By Corollary 1 this means that system (2) has no driver of $x$-symmetreis of zero order mutually independent with $\sigma_{1}=\left(u_{x}^{1}, u_{x}^{2}\right)^{\top}$. Thus, drivers (3) define the basis set for this system.

In work [11], for system (2), there were constructed the drivers of the symmetries

$$
\begin{aligned}
& S_{1}=\left(\begin{array}{c}
u_{x}^{1} \\
0
\end{array}\right) D_{x}+\left(\begin{array}{c}
u_{x x}^{1}+\frac{u_{x}^{1} u_{x x}^{2}}{u_{x}^{2}}-\frac{2 u^{1} u_{x}^{1} u_{x}^{2}+u^{2}\left(u_{x}^{1}\right)^{2}}{u^{1} u^{2}+c} \\
\frac{u^{2} u_{x}^{2} u_{x}^{1}}{u^{1} u^{2}+c}
\end{array}\right), \\
& S_{2}=\left(\begin{array}{c}
0 \\
u_{x}^{2}
\end{array}\right) D_{x}+\left(\begin{array}{c}
2 \frac{u^{1} u_{x}^{1} u_{x}^{2}+u^{2}\left(u_{x}^{1}\right)^{2}}{u^{1} u^{2}+c}-2 \frac{u_{x}^{1} u_{x x}^{2}}{u_{x}^{2}}-u_{x x}^{1} \\
-u_{x x}^{2}
\end{array}\right) .
\end{aligned}
$$

By Theorem 1, they can be expressed in terms of the basis drivers of symmetries $\sigma_{1}$ and $\sigma_{2}$ defined by formula (3). Applying the approach used in the proof of Theorem 1, we obtain

$$
S_{1}=\sigma_{2} \circ \omega, \quad S_{2}=\sigma_{1} \circ\left(D_{x}-\tilde{\omega}\right)-\sigma_{2} \circ \omega,
$$

where $\omega=u_{x}^{1} u_{x}^{2} /\left(u^{1} u^{2}+c\right), \tilde{\omega}=u_{x x}^{2} / u_{x}^{2}-\left(u^{2} u_{x}^{1}\right) /\left(u^{1} u^{2}+c\right)$. A straightforward checking shows that $\omega$ and $\tilde{\omega}$ belongs to the kernel of $D_{y}$.

Example 2. We denote by $\mathbf{e}^{\mathbf{u}}$ the vector $\left(\exp \left(u^{1}\right), \exp \left(u^{2}\right), \ldots, \exp \left(u^{n}\right)\right)^{\top}$. Then we can write system (6) as $u_{x y}=A \mathbf{e}^{\mathbf{u}}$, where $A$ is a constant matrix of size $n \times n$. For further arguing it is also convenient to denote by $\{z\}$ the diagonal matrix with the diagonal formed by the coordinates of the vector $z$ :

$$
\{z\}:=\operatorname{diag}\left\{z^{1}, z^{2}, \ldots, z^{n}\right\} .
$$

In view of this notation we have $Y_{1}=H_{1}=A\left\{\mathbf{e}^{\mathbf{u}}\right\}$. This is why in the general situation (as $\operatorname{det}(A) \neq 0$ ) system $(6)$ has no drivers of $x$-symmetries of zero order.

By (8) we can find easily $a_{1}=-A\left\{u_{y}\right\} A^{-1}$ and then by formula (9) we calculate $H_{2}=$ $H_{1}-A\left\{A \mathbf{e}^{\mathbf{u}}\right\} A^{-1}$ and

$$
Y_{2}=H_{2} H_{1}=A\left\{\mathbf{e}^{\mathbf{u}}\right\} A\left\{\mathbf{e}^{\mathbf{u}}\right\}-A\left\{A \mathbf{e}^{\mathbf{u}}\right\}\left\{\mathbf{e}^{\mathbf{u}}\right\}=A C\left\{\mathbf{e}^{\mathbf{u}}\right\},
$$

where $C=\left\{\mathbf{e}^{\mathbf{u}}\right\} A-\left\{A \mathbf{e}^{\mathbf{u}}\right\}$. We denote by $\tilde{C}$ the matrix of size $(n-1) \times(n-1)$ obtained by removing the last row and the last column in the matrix $C$. It is easy to see that

$$
\operatorname{det}(\tilde{C})=(-1)^{n-1} \exp \left((n-1) u^{n}\right) \prod_{i=1}^{n-1} A_{n}^{i}+\ldots,
$$


where the dots stand for the terms not involving $\exp \left((n-1) u^{n}\right)$, namely, involving $\exp \left(u^{n}\right)$ in powers less than $n-1$. This is why the matrix $\tilde{C}$ is non-degenerate and $Y_{2}$ has the rank $(n-1)$ if $\operatorname{det}(A) \prod_{i=1}^{n-1} A_{n}^{i} \neq 0$. Thus, in the general case system (6) possesses the unique (up to the right multiplication by a scalar function in ker $D_{y}$ ) driver of $x$-symmetries of the first order.

\section{BIBLIOGRAPHY}

1. A.V. Zhiber, A.B. Shabat. Klein-Gordon equations with a nontrivial group // Dokl. Akad. Nauk SSSR. 247:5, 1103-1107 (1979). [Sov. Phys. Dokl. 24:8, 607-609 (1979).]

2. A.N. Leznov, A.B. Shabat. Truncation conditions for perturbation theory series // in "Integrable Systems", Bashkir Branch, Acad. Sci. USSR, Ufa, 34-44 (1982). (in Russian).

3. A.N. Leznov, V.G. Smirnov, A.B. Shabat. The group of internal symmetries and the conditions of integrability of two-dimensional dynamical systems // Teor. Matem. Fiz. 51:1, 10-21 (1982). [Theor. Math. Phys. 51:1, 322-330 (1982).]

4. D.K. Demskoi. One Class of Liouville-type systems // Teor. Matem. Fiz. 141:2, 208-227 (2004). [Theor. Math. Phys. 141:2, 1509-1527 (2004).]

5. S.Ya. Startsev. Cascade method of Laplace integration for linear hyperbolic systems of equations // Matem. Zametki. 83:1, 107-118 (2008). [Math. Notes. 83:1, 97-106 (2008).]

6. F.G. Tricomi. Lectures on partial differential equations. Inostr. Liter., Moscow (1957). [Lezioni sulle equazioni a derivate parziali. Editrice Gheroni, Torino (1954). (in Italian).]

7. A.V. Zhiber, V.V. Sokolov. Exactly integrable hyperbolic equations of Liouville type // Uspekhi Matem. Nauk. 56:1, 63-106 (2001). [Russ. Math. Surv. 56:1, 61-101 (2001).]

8. A.M. Guryeva, A.V. Zhiber. Laplace invariants of two-dimensional open Toda lattices // Teor. Matem. Fiz. 138:3, 401-421 (2004). [Theor. Math. Phys. 138:3, 338-355 (2004).]

9. A.M. Guryeva, A.V. Zhiber Laplace invariants of Toda lattices with the exceptional Cartan matrices // Preprint: arXiv:nlin/0512001 [nlin.SI], (2005).

10. A.V. Zhiber, S.Ya. Startsev. Integrals, solutions, and existence Problems for Laplace transformations of linear hyperbolic systems // Matem. Zametki. 74:6, 848-857 (2003). [Math. Notes. 74:6, 803-811 (2003).]

11. V.V. Sokolov, S.Ya. Startsev. Symmetries of nonlinear hyperbolic systems of the Toda chain type // Teor. Matem. Fiz. 155:2, 344-355 (2008). [Theor. Math. Phys. 155:2, 802-811 (2008).]

Sergey Yakovlevich Startsev,

Institute of Mathematics,

Ufa Federal Research Center, RAS,

Chernyshevsky str. 112,

450008, Ufa, Russia

E-mail: startsev@anrb.ru 